\title{
L'adolescent, la différence et le lien social
}

Rajaa Stitou

L'auteur montre que si l'adolescent questionne autant la famille et la civilisation moderne dans leur rapport aux idéaux, c'est parce qu'à travers lui se repose avec acuité la question de la différence, interpellant l'autre au cœur de ce qui le fonde et le divise, à la croisée $d u$ singulier et du collectif. Cette différence qui se ravive à travers l'épreuve de grandir et qui s'exacerbe dans le contexte de l'immigration peut être constructive lorsqu'elle est reconnue comme porteuse d'altérité. Mais elle peut déboucher sur la ruine lorsqu'elle est perçue comme une menace et qu'elle n'est plus entourée par des repères symboliques. C'est ce dont témoigne l'expérience clinique au quotidien auprès d'un bon nombre d' adolescents dit en rupture de lien social, qui finissent par se confondre avec l'image déficitaire que leur renvoi d'eux même l'Autre Social et/ou parental.

Mots clés: Adolescence, différence, lien social et familial, reconstruction/déconstruction 
La pratique clinique auprès d'adolescents dits "en rupture de lien social” constitue le point de départ de ce cheminement réflexif qui m'amène à prendre en compte le contexte sociétal et ce qui sous tend ses modes d'altérité, dans leur versant constructif mais aussi à travers les impasses. En effet, les souffrances subjectives contemporaines ne cessent de secouer les repères collectifs. Nos dispositifs d'écoute ne peuvent ignorer ces effets de civilisation. L'hypothèse qui guide ma démarche, c'est que si l'adolescence questionne autant la civilisation moderne dans son rapport à ses idéaux, c’est parce qu’à travers elle se repose de manière cruciale la question du lien social dans son rapport à la différence. En effet, l'adolescence est actuellement désignée par le discours commun comme une adolescence à risque, venant bouleverser ce qui unit l'humain collectivement. Ce sentiment de crainte est nourri par l'accroissement de l'hétérogène du fait de l'immigration qui vient mettre à l'épreuve l'altérité. Pourtant, par-delà l'invention récente du mot adolescence, la jeunesse a toujours constitué le révélateur de démêlés de la société avec elle-même, quelles que soient les cultures et les époques. De tout temps, elle a été source d'inquiétude interpellant l'autre au cœur de ce qui le fonde, le divise et le différencie, à la croisée du singulier et du collectif. En effet, la société a toujours construit des montages afin de border l'angoisse engendrée par ces moments de passage qui actualisent ce qui est au principe même de l'existence du sujet dans son articulation au lien social, c'est-à-dire l'impossible identité de ce que je suis, d'où je viens et où je vais. Cet insaisissable qu'incarne l'adolescent et qui se redouble avec la différence 
culturelle, n'est-ce pas ce qui provoque dans le contemporain de la civilisation la survenue de fantasmes tentant de donner forme à ce qui se présente comme étranger tout en restant dans une familiarité troublante?

Dans les sociétés dites traditionnelles, la puberté est considérée comme une période d'incertitude qui expose à tous les dangers, d’où la mobilité de toute collectivité qui autorise l'enfant grandissant à traverser un temps d'errance et d'indécision de l'appartenance, impliquant une nouvelle identification sexuée, ainsi qu'une ré-appropriation du lien aux autres. Après ce temps d'errance reconnu par la société, qui permet par là même au jeune de se reconnaître à la fois différent, et semblable aux autres, intervient le temps d'agrégation (Van Gennep A., 1909) qui constitue une reconsécration identitaire. Parents et proches sont amenés à déplacer leur regard sur l'adulte en devenir en lui donnant une autre place, à partir de nouvelles limites inscrivant un nouvel ordre d'échanges.

Le rite initiatique est donc consacré à la fois au jeune et à son entourage car grandir n’est pas une affaire privée mais un événement à partager, inscriptible dans le lien social, qui permet à la collectivité de se rassembler autour de ce qui demeure impensé afin de le métaphoriser.

Cela est vrai pour les sociétés traditionnelles, mais cela est vrai aussi pour les civilisations modernes. Qu'il soit traditionnel, modernisé, abrégé, ou réformé, le rite demeure une nécessité quant à son fondement. Il tient à distance le chaos et la pulsion de mort lorsqu'il ne se fige pas dans ses usages au détriment de sa dimension symbolique. À travers lui, se tisse et se reconstruit le lien social. Cela nous conduit à faire un détour en reconsidérant ce qui lie les hommes les uns aux autres à partir de ce qui fonde et articule ce lien.

\section{Le lien social, son fondement et sa nécessité}

Avec Totem et tabou, Freud (1912) nous montre que le fondement du lien social est tributaire d'un acte de séparation. Le lien social, nous dit-il, naît, se tisse et s'incarne dans un drame fondateur, celui du meurtre du père de la horde à travers lequel s’évoque le lieu de la confusion, de la négation de l'altérité. C’est donc la coupure d'avec le chaos de la horde sauvage qui instaure le premier acte convertissant l'impossible en un possible lien. C'est depuis ce meurtre primordial que s'accomplit le renouvellement de ce qui va du crime à la Loi et aux lois qui répondent à une nécessité: protéger, humaniser, lier les hommes entre eux, lien qui ne peut s'établir qu'au prix d'un renoncement.

En effet, dans l'après-coup du crime, les hommes découvrent leur culpabilité et par là même leurs failles subjectives les situant hors tout savoir, tout pouvoir, 


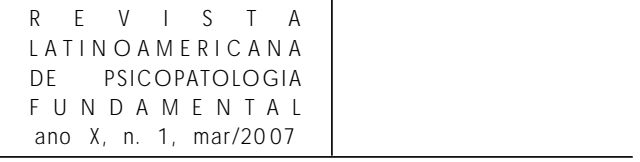

tout avoir. Ils réalisent que la jouissance absolue, en barrant l'accès à la parole, ne peut déboucher que sur la ruine. D’où leur acceptation d’une sexualité qui se refuse à une pleine satisfaction. Un pacte va instaurer le partage des femmes dans l'exogamie. Ce partage leur permet de s'inscrire dans le lien social mais aussi dans un lien de filiation, de non-confusion des générations les situant comme les héritiers d'un legs venu d'ailleurs, d'une mémoire marquée par le refoulement.

C'est ainsi qu'advient l'antériorité de l'Autre, que se noue la dette au don et que se présentifie la différence sans laquelle aucun échange, aucune transaction n'est possible. C'est ainsi que le père de la toute puissance devient le père symbolique, père dont on peut dire qu'il est mort depuis toujours car le symbolique réorganise le passé après coup. L’inscription de l'homme dans le lien social peut aussi être considéré comme une inscription dans la parole. Subjectivité, parole et lien social surgissent là où s'effectue la séparation d'avec la horde. Le meurtre du père primitif en tant qu'exil ou coupure d'avec la horde originelle ouvre ainsi sur la Loi Universelle de l'interdit de l'inceste, Loi que chaque culture va instituer et dont chaque sujet va se soutenir

Or, il ne peut être d'interdits sans transgression, pas plus que de symbole sans imaginaire ou de mémoire sans déformation; d’où la nécessité des tabous et de leurs rites conjuratoires qui, à chaque crise occasionnelle, à chaque deuil ou à chaque génération perpétuent le pacte symbolique qui lie les hommes les uns aux autres. À travers ce pacte qui rappelle à chaque sujet sa condition d'être parlant, mortel et sexué se rejoue l'accès de l'homme à la culture. Mais le lien social nous renvoie également aux liens familiaux et à ce qu'ils impliquent pour tout un chacun.

\section{Les liens familiaux}

Les liens familiaux s’originent également dans une séparation issue de l'incomplétude originelle et du refoulement, inscrivant l'humain dans une antécédence toujours Autre, un ordre symbolique préexistant faisant advenir la mémoire comme une antériorité à venir. Tout sujet est fille ou fils de... Il est pris dans une généalogie, un réseau signifiant. La famille est le lieu où se fonde le lien à l'Autre et aux autres. Sa fonction est de permettre à l'enfant de s'inscrire dans ses premières identifications. Mais la famille ne constitue pas le seul modèle identificatoire; les références culturelles d’une société offrent à l'enfant des figures mythiques ou héroïques à travers lesquelles il peut trouver un appui. En tant que médiateur entre le groupe qui a apporté les premiers soins au petit d'homme du fait de sa prématuration et la société, la famille constitue le lieu où s’opère la socialisation des pulsions. Freud nous a appris que c'est la loi œdipienne 
qui constitue le principe de cette régulation, et J. Lacan (1938) a fait du langage dans "Les complexes familiaux" la spécificité de la nature humaine. En effet, ce qui distingue l'être humain de l'animal, ce sont les règles d'alliance qu'une loi régule et à partir de laquelle se noue à travers les générations le fil des lignées.

Ces nouages familiaux, qui ont une configuration différente en fonction des cultures et des époques, obéissent tous au même principe qui est au cœur de toute société: l'interdit de l'inceste. Ce que transmet la famille, c'est la loi mais aussi le nom, ce qui permet de différencier sexe et génération. En ce sens, la famille constitue un truchement qui permet l'entrée de l'enfant dans le monde de la représentation symbolique, ce qui s'effectue non sans ratage en fonction de la structuration de tout un chacun et des relations que son entourage noue avec lui.

Mais dans toute représentation, il y a de l'irreprésentable, un ailleurs qui n'a pas de figure, mais demeure le lieu d'un appel. Cet irreprésentable, cet innommable inscrit au cœur même de toute nomination et qui échappe aux désirs parentaux est à l'origine du langage mais il est aussi articulé à l'institution familiale. Il instaure une discontinuité dans la transmission qui fait que l'enfant n'est pas le simple duplicata de ses parents. C'est à partir de là qu'une histoire nouvelle peut toujours advenir.

Cette discontinuité présentifie un écart, une béance que redécouvre l'enfant grandissant et/ou ses parents dans les épreuves de leur existence, les confrontant ainsi à leur incomplétude, ce qui nécessite un recours à la fiction, un passage par l'Autre afin d'apaiser l'insoutenable du réel. Autrement dit, les liens qui articulent le groupe familial ne cessent de se déconstruire et de se reconstruire en fonction des séparations et des remaniements qui scandent le mouvement même de son devenir: naître, grandir, devenir parent ou grand parent, vieillir, partir.

L’adolescence qui se situe à la croisée du singulier et du social peut être considérée comme le paradigme de cette déconstruction/reconstruction. Il s’agit là d’une expérience psychique, d'une mobilité subjective à travers lesquelles se repose d'une manière Autre la question de la séparation et du lien, de l'appartenance et du destin, de la mort et du sexuel. En effet tout adolescent doit affronter le surgissement de l'inconnu, traverser de nouveau la coupure qui le constitue afin de s'arrimer avec sa singularité à la communauté des adultes. Cela ne va pas sans vacillation, aussi bien pour l'enfant grandissant que pour l'entourage qui se trouve devant la nécessité d'en repasser par des opérations de symbolisation afin d'engager le passé sur la scène de l'avenir, de trouver des réaménagements nécessaires au tissage de nouveaux liens à partir d'une dialectique constructive entre continuité et discontinuité. C'est ainsi que l'adolescent peut s'inscrire dans l'altérité, trouver sa place dans le monde et s'autoriser à inventer, c'est-à-dire à ne pas se figer dans l'imitation servile de ses parents ou des modèles sociaux. 


\section{$\begin{array}{lllllll}R & E & V & \text { I } & S & T & A\end{array}$ \\ LATIN O AMERICAN A \\ DE PSICOPATO LOGIA \\ F U N D A M E N T A L \\ ano $X$, n. 1, mar/2007}

Mais pour que l'adolescent s'ouvre à cette dialectique, la famille ainsi que la société, à travers les mythes et les rites qui s’y rattachent doivent êtres porteurs de repères symboliques.

Selon comment ces derniers s'inscrivent, l'adolescent peut se trouver en place de répondre à ce qu'il y a de symptomatique dans les structures familiales et sociales. Ces situations peuvent s'exacerber dans le contexte de l'immigration au point de déstabiliser le lien social. La différence à partir de laquelle se détermine le "nous" du collectif s'exacerbe, voire se redouble face à l'adolescent issu d'ailleurs, au point de ne plus être reconnue comme porteuse d'altérité mais comme une menace à congédier ou à intégrer au même.

Or qu'en est-il lorsque le passage adolescent est perçu par l'Autre parental comme une menace? Qu'en est-il lorsque la société massifie l’adolescent ou bien fétichise sa différence culturelle au détriment de sa singularité? Les nombreuses appellations qui désignent ces jeunes en témoignent: “jeunes de banlieue”, “fils d’immigrés de troisième ou quatrième génération”, “enfants du déracinement”... Cette massification solidaire d'un rejet de l'Autre et par là même de toute étrangeté ne contribue-t-elle pas à nourrir toutes formes de défense mortifiante: brandir sa différence à l'extrême ou au contraire la nier au point de faire taire le désir et la singularité.

L'expérience clinique nous montre que bon nombre de jeunes dits en rupture de lien social finissent par se confondre avec l'image déficitaire que leur renvoie d'eux-mêmes la société contemporaine de plus en plus soucieuse de normativité et d’homogénéité. En se regroupant entre mêmes d’une manière identifiée, identique, ces jeunes se mettent en avant en tant que représentants de leur communauté et non pas à partir de ce qui les particularise subjectivement. Le phénomène de bandes bien connu chez les adolescents peut certes constituer un étayage, un support identificatoire entre “pairs” dans cette période de recherche d'un nouveau lien encore informe, non élaboré, mais lorsque l'identification est une identification à l'autre comme même, lorsqu'elle n'ouvre plus sur du tout Autre, la bande se transforme alors en ghetto. Le lien qui l'a réunie devient lien de contagion. Les jeunes se sédentarisent ainsi dans le miroir de la violence qui fait d'eux des êtres tous pareils ou complètement à part.

Certains parents expatriés redécouvrent à l'adolescence des enfants une nouvelle “épreuve de l'étranger” qui les renvoie non seulement au vécu de l’immigration mais aussi à l'exil ${ }^{1}$ qui les fonde. L'enfant qui a grandi leur échappe, il de-

1. L'exil n'est pas réductible au déplacement géographique; Il renvoie à la séparation fondatrice du sujet et de l'altérité, séparation qui se ravive avec le changement de pays et de culture. 
vient étranger. Plusieurs parents nous demandent d'ailleurs d'aider leur fils ou leur fille à redevenir comme avant à l'image de l'enfant merveilleux, inchangé et inchangeable, sans lignage, sans lignée. Cela ne va pas sans angoisse. Et c’est souvent à cette période qu'ils imposent à leur progéniture le retour au pays d'origine. C'est ce que nous proposons d'examiner à travers quelques fragments cliniques.

\section{Fragments cliniques}

Aïcha est une adolescente d'origine turque âgée de quinze ans. Devenue méconnaissable au dire de ses parents, elle aurait déstabilisé toute la famille par ses conduites étrangères aux mœurs et aux lois de leur pays (sorties fréquentes, extravagances...). Cette non-conformité à ce que leurs traditions déterminent comme "être femme" les inquiète. Mais Aïcha dira la même chose de ses parents en insistant sur cet insoutenable sentiment d’étrangeté: “Je ne les comprends pas. Tout un monde nous sépare. Je respecte leur mentalité, mais je ne suis pas comme eux.” Cette adolescente qui m’a été adressée par un médecin a fait une tentative de suicide quelques mois après avoir fugué. Craignant que la menace du père (retour dans le pays d'origine) ne soit mise à exécution, Aïcha a entre temps demandé l'aide d'une assistante sociale. Affectée par cette demande, l'assistante sociale a donné raison à Aïcha et a entrepris les démarches nécessaires pour un placement en foyer. Mais Aïcha s’est rétractée, ne supportant pas l’idée de voir sa famille malheureuse. Les parents, contactés par le juge, se sont effondrés. C’est dans cette atmosphère qu'Aïcha a tenté de mettre fin à ses jours.

C'est dans l'agir, un agir symétrique à celui de la famille et de l'assistante sociale qu'Aïcha tente d'échapper au tiraillement entre le vœu de maintenir les liens avec ses parents et la tentative d'affirmer sa singularité. L'incertitude propre à l'adolescence que vient ici redoubler la dimension culturelle provoque une angoisse qui crée un appel au nom-du-père mais qui ne rencontre en guise de réponse qu'un discours objectivant qui la met de côté en tant que sujet. Les passages à l'acte de Aïcha (fugue, tentative de suicide) peuvent être entendus comme une tentative de s'expulser du lieu que les autres lui imposent, ou dans lequel ils tentent de la ramener. Elle ne se sent pas à sa place.

D'un côté ses parents exigent le retour au même (à travers le retour aux origines, la Turquie) face à l’insupportable étrangeté que leur renvoie leur fille. C’est le modèle occidental qui selon eux les dépossède de leur enfant.

D’un autre côté, en répondant au patent de la demande de Aïcha, et pensant bien faire, l'assistante sociale désapprouve les "signifiants parentaux" (Hassoun, 1994) de l'adolescente et lui propose un autre lieu d'assimilation factice. 


\section{$\begin{array}{lllllll}R & E & V & \text { I } & S & T & A\end{array}$ \\ LATIN O AMERICAN A \\ DE PSICO PATO LOGIA \\ F U N D A M E N T A L \\ ano $X$, n. $1, \operatorname{mar} / 2007$}

Des deux côtés, Aïcha est assignée à une place qui n’est pas la sienne, mais celle d'un fantasme de complétude ou d'un conformisme qui l'empêche d'en passer par l'Autre, et du coup l'empêche d'occuper une position de sujet à partir de l'épreuve de castration.

La mise en avant de la différence culturelle vient occulter la division subjective et imposer quelque chose de l'ordre de la fixité et non pas de la mobilité. L'épreuve de l'étranger n'est pas vécue comme un événement faisant partie de l'histoire du sujet mais comme un événement extérieur, ne concernant en rien la division d'avec soi-même, division à la base de tout sentiment d'identité.

Or, ce que réclame Aïcha, c'est d’être reconnue dans sa singularité afin de se reconnaître elle-même et d'affronter les autres; car comment affronter l'autre qui ne me reconnaît pas ou qui perçoit en moi une menace, un danger?

Aucune parole consistante ne semble soutenir ce temps de passage dans lequel cette adolescente ne trouve plus ses marques, faute d’être acceptée avec ce savoir énigmatique qui l'habite à son insu. L'entourage lui impose au contraire de rester sédentaire dans ses difficultés. "Il fallait que ça déménage” dira d’ailleurs Aïcha. Cette non-reconnaissance peut être ravageante.

“Je n'ai pas peur de mourir dira encore Aïcha, ce qui me tue, c’est le regard de mes parents”. Lorsque le regard de l'Autre devient persécuteur, lorsqu'il n’engage pas le désir, il peut conduire à la perte de ce qui fait tenir debout le sujet. Ce regard de l'Autre qui permet à l'enfant au moment du miroir de rassembler les morceaux épars grâce à la nomination et à la présentification du manque, et qui est reconvoqué à l'adolescence s'avère problématique pour Aïcha. À travers lui, elle se sent dénigrée en tant que femme en devenir. Cette question du féminin a d’ailleurs été posée dès sa naissance. Son père aurait voulu un garçon.

Tantôt fuyante, tantôt s’agrippant à mon regard, comme pour vérifier sa consistance à mes yeux, Aïcha exprimera tout au long de notre première rencontre son sentiment d’être désorientée: “Si j’étais un garçon ou une française, je n’aurais pas vécu ce calvaire."

C'est autour d'un bracelet que je portais ce jour-là que s'est nouée la relation. Attirée par cet objet dont les motifs “orientaux” la renvoient à quelque chose “d'extime”, Aïcha a pu interroger ce qu'il en est à la fois de son appartenance culturelle et de sa position féminine.

Adil: Tout autre est la problématique d'Adil.

Il est âgé de dix-sept ans. Dernier et unique garçon d'une fratrie de cinq enfants, il me raconte qu'il est issu d'une famille kabyle qui a émigré en France dans les années soixante. Scolarisé en classe de première, son parcours d'étudiant s’est situé jusque-là dans la moyenne, mais, depuis quelques mois, période où son père a pris sa retraite, tout a basculé dit-il. Ses conduites déviantes inquiéteraient 
son entourage. Ça devient invivable avec ses parents, il ne supporte aucun reproche. Ses professeurs se plaignent de son absentéisme et de son agressivité, et l'accusent d'absorber des toxiques.

Je verrai Adil une fois à la demande du psychiatre du Centre Médico-PsychoPédagogique, suite à une menace d'expulsion du lycée, et une autre fois à sa demande, avant de rencontrer sa mère.

\section{Rencontre avec Adil}

Pendant le premier entretien, Adil est très anxieux, mal à l'aise dans son corps et peu coopérant. Ce n'est que lors de la deuxième rencontre qu'il accepte de se confier malgré sa difficulté à s'exprimer. Un léger sourire s’esquisse lorsque je lui demande ce que représente pour lui l'ancre tatouée sur son épaule. “C'est un symbole marin que j'ai choisi au hasard.” Il reconnaît fumer du haschich de temps en temps, mais ce n'est sûrement pas ce qui lui fait du mal. Ce qui lui fait mal, c’est sa famille “qui lui prend la tête”, en particulier sa mère et ses grandes sœurs qui contrôlent tout: “Je n’ai aucun droit à la parole. Quand je dis quelque chose, j'ai tort. Quand j'écoute de la musique, c'est trop fort, quand je sors avec mes copains, c'est des voyous."

Il s'inquiète pour son père qui “débranche” et ne supporte pas le fait de le voir malade. "Ma mère lui prend trop la tête, elle devrait le laisser partir en Algérie. Il n'a pas envie de finir sa vie ici et c'est son droit”. Adil interroge alors le lien d'une mère possessive à son père réduit à n'en être que l'objet de son caprice, ce à quoi lui se refuse. Du pays d'origine de son père, il ne sait rien à part la musique "raï" et les plats que cuisine sa mère. Il se dit étranger à ce monde dont il ne connaît que les images de guerre et de ruine, celles que rapportent les journaux mais aussi celles que rapporte son oncle paternel. En France aussi Adil se considère comme un étranger: "La nationalité française ne change pas grandchose, les remarques racistes me le rappellent tous les jours.” Mais étranger, il l'est aussi à lui-même puisqu'il dit ne pas se reconnaître dans ses accès de colère. Sa violence témoigne de ce qui ne parvient pas à se symboliser.

La seule façon pour Adil de s'en sortir, c'est de quitter la cité dans laquelle il habite et le milieu familial. Il souhaite aller en internat, mais ne sait pas comment convaincre sa mère.

Ce déplacement reporté dans l'espace, au niveau de la géographie afin d'échapper à l'enclos familial, je l'ai aussi entendu comme un mouvement subjectif, une tentative d'aller vers son désir afin d'effectuer son entrée dans ce temps où se dessinent pour les jeunes de son âge d'autres valeurs. 


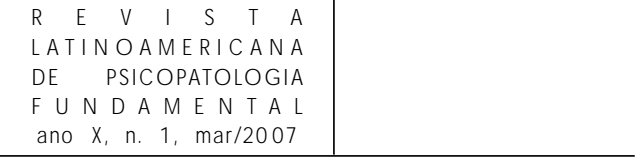

Ce temps de rencontre à travers lequel j'ai occupé le lieu d'une adresse a consisté à accueillir le “ce qui ne va pas” sur quoi s’appuie la demande du sujet, en laissant place au manque afin que la plainte d'Adil évolue vers un questionnement qui lui donne la possibilité de partir à la découverte de lui-même en s'impliquant en tant que sujet de sa propre histoire.

\section{Madame B.}

Mme B. n'accepte pas la métamorphose de son fils. Il est devenu l'étranger qui ne revient plus au même. La singularité de ce fils qui a grandi est vécue par elle comme une dérobade.

“C'est mon fils unique et j'espérais tellement qu'il soit un homme, qu'il réussisse dans la vie ”. Elle insiste longuement sur la relation harmonieuse qui les liait lorsqu'il était enfant. Il incarnait l'enfant rêvé. Il avait tout pour être heureux et pour la rendre heureuse. Les troubles du sommeil, et l'énurésie nocturne qui a duré jusqu’à l'âge de neuf ans n’ont jamais fait énigme pour elle. Ils seront considérés comme des détails.

Tantôt l'accusant d'avoir changé, tantôt le posant comme victime de la jalousie de ses sœurs et de l'indifférence de son père, Mme B. parlera de son fils comme s'il s'agissait de son double. Le rejet dont il est l'objet, c'est ce qui, selon elle, les rapproche, les lie l'un à l'autre. Elle aussi a été rejetée par sa famille. Elle avait le même âge que Adil lorsque ses parents l'ont obligée à se marier avec un homme plus âgé qu'elle et à s'exiler. “Je n’ai pas pu poursuivre mes études et je n’ai pas vécu de vie de couple” précise-t-elle. “Mon mari ne s’intéressait qu’à son travail, et à présent il ne souhaite qu’une chose: mourir en Algérie. Mais je refuse de m'exiler de nouveau et je refuse de quitter mes enfants. J'ai sacrifié toute ma jeunesse pour eux et j'ai tout fait pour qu'ils ne subissent pas ce que j'ai subi.”

La demande manifeste de cette mère est que son fils réussisse dans la vie, mais ce qui s’y évoque implicitement, ne pourrait-on pas le formuler de la manière suivante: “Reste mon objet, sinon j'en mourrai?”. N'est-ce pas ce que Adil, qui tente à travers ses passages à l'acte de sortir de la jouissance de l'endogamie, a entendu?

Mais Mme B. refuse tout changement qui est vécu par elle comme la manifestation d'un insupportable. Elle souhaite que son fils continue à être l'objet de sa complétude, c'est-à-dire qu'il ne demande rien. Toute demande passe par la médiation de l'Autre. C'est ainsi que le désir est pacifié. Mais lorsque cette médiation est en faillite, lorsque le rapport à l'Autre est obstrué, la demande se rabat sur le besoin, le sujet n'a plus rien à demander, il exige tout dans l'immédiat. 
À quoi de radicalement étranger Mme B. est-elle confrontée à travers l'adolescence de son fils? Telle est la question que je me suis entendue dire, question qui a eu l'effet d'un dessaisissement, libérant du coup la parole de toute maîtrise, de toute emprise.

Cette question qui a fait aussi énigme pour elle a pu ainsi être conviée afin d'être reprise dans les défilés du signifiant. Ce n'est qu'à partir de là qu'un travail d'élaboration a pu se mettre en place.

Ces différents fragments cliniques nous montrent que grandir constitue une véritable épreuve à la croisée du singulier et du collectif, qui se complexifie dans le contexte de l'expatriation au point où se fragilise parfois la frontière entre le réel et l'imaginaire, entre la scène privée, fantasmatique et la scène sociale. Cette épreuve qui nécessite un déplacement aussi bien pour l'enfant grandissant que pour son entourage, peut être structurante lorsqu'elle parvient à se métaphoriser, lorsqu'elle parvient à ouvrir sur l'altérité. Mais elle peut aussi conduire au "nulle“ part lorsqu'elle est vécue comme quelque chose de dramatique voir de catastrophique non étayée par une parole constructive... Tels sont les éléments à prendre en compte dans l'écoute clinique face aux adolescents dit "en rupture de lien social”; clinique qui se conjugue au singulier, mais sans couper le sujet de ses références culturelles ou de la civilisation qu’il traverse.

Références

Freud, Sigmund (1909). Le roman familial. In: Névrose, psychose et perversion. Paris: PUF, 1974. p. 157-60.

(1912). Totem et tabou. Paris: Payot, 1984.

FÉDIDA, Pierre. Le site de l'étranger. Paris: PUF, 1995.

GutTon, Philippe. Le pubertaire. Paris: PUF, 1991.

Hassoun, Jacques. Les contrebandiers de la mémoire. Paris: Syros, 1994.

LaCAN, Jacques (1938). Les complexes familiaux. Paris: Navarin, 1984.

Deux notes pour l'enfant. Ornicar?, Paris, n. 39, p. 13-4, 1969.

Legendre, Pierre. L'inestimable objet de la transmission. Paris: Fayard, 1992.

LÉvi-Strauss, Claude (1949). Les structures élémentaires de la parenté. Paris: PUF, 1967.

RAssial, Jean-Jacques J. Le passage adolescent. De la famille au lien social. Ramonville Saint-Agne: Érès, 1996. 


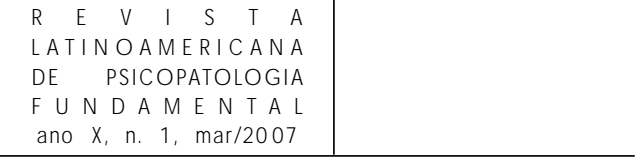

Stiтou, Rajaa. Universalité et singularité de l’exil. Psychologie clinique, Paris, n. 3, p. 13-20, 1997.

VAN Gennep, Arnold. Les rites de passage. Paris: Nousry, 1909.

\section{Resumés}

O autor mostra que, se o adolescente questiona tanto a família e a civilização moderna nas suas relações com os ideais, é porque através dele se fixa com acuidade a questão da diferença, interpelando o Outro no centro do que lhe funda e que lhe divide, no cruzamento do singular e do coletivo. Essa diferença, que se reanima através da experiência do crescimento e que se exacerba no contexto da imigração, pode ser construtiva quando reconhecida como portadora de alteridade. Mas ela pode resultar na ruína quando é percebida como uma ameaça e quando não é acompanhada de referências simbólicas. Isso é o que testemunha a experiência clínica cotidiana com um bom número de adolescentes considerados em rompimento com o vínculo social, que acabam se confundindo com a imagem deficiente deles mesmos que lhes remetem o Outro social e/ou parental.

Palavras-chave: Adolescência, diferença, vínculo social e familiar, reconstrução/deconstrução

El autor muestra que si el adolescente cuestiona tanto la familia y la civilización moderna en relación a los ideales, es porque a través de él reaparece intensamente la cuestión de la diferencia, cuestionando al otro en el centro de lo que lo funda y lo divide, en el cruce de lo singular y de lo colectivo. Esta diferencia que se reaviva a través de la experiencia del crecimiento y que se exacerba en el contexto de la inmigración, puede ser constructiva cuando se la reconoce como portadora de alteridad. Pero puede desembocar en fracaso cuando se la percibe como una amenaza y no es acompañada de referencias simbólicas. Es lo que testimonia la experiencia clínica cotidiana con un buen número de adolescentes considerados en ruptura de lazo social, que terminan por confundirse con la imagen deficitaria de si mismos que el Otro Social y/o parental les devuelve.

Palabras claves: Adolescencia, diferencia, lazo social y familiar, reconstrucción/ deconstrucción

This paper broaches the discomfort caused by adolescents both in their families and in the relationship of modern civilization with ideals. This would seem to be because they adeptly make differences rise to the surface, and confront the Other at the center of his or her foundation and divisions, at the cross point between the singular 


\section{ARTIG OS}

and the collective. This difference, which is stimulated by the growth process and exacerbated in the context of immigration, can be constructive when it is recognized as a bearer of otherness. But it can result in disaster when perceived as a threat and when no longer surrounded by symbolic references. This can be seen in daily clinical practice with many teenagers considered to be in rupture with social bonds and who take on a deficient self-image that has been passed on to them by the social and/or parental Other.

Key words: Adolescence, disparity, social and family ties, deconstruction/reconstruction 